\title{
Did the COVID-19 Pandemic Have Immediate Impacts on Japanese Children? Evidence of the Socio-Emotional Behavior and Digital Skills
}

Yusuke Moriguchi ( $\nabla$ moriguchi.yusuke.8s@kyoto-u.ac.jp )

Kyoto University https://orcid.org/0000-0002-9002-7834

\section{Chifumi Sakata}

Kyoto University

\section{Xianwei Meng}

Doshisha Daigaku

Naoya Todo

Tsukuba Daigaku

\section{Research Article}

Keywords: COVID-19, pandemic, child development, socio-emotional skill, digital skill

Posted Date: August 11th, 2020

DOI: https://doi.org/10.21203/rs.3.rs-55554/v1

License: (c) (i) This work is licensed under a Creative Commons Attribution 4.0 International License. Read Full License 


\section{Abstract}

Background: A novel coronavirus, SARS-CoV-2, has spread widely throughout the world. To reduce the spread of infection, children are prevented from going to school and have fewer opportunities for inperson communication. Although the pandemic has affected the everyday lives of children, its impact on their development is unknown. This cross-sectional study compared Japanese children's socio-emotional behaviors and skills for operating digital devices before and during the pandemic.

Methods: Parents completed a web-based questionnaire before and during the pandemic for children aged 0-9. Children's socio-emotional development in the context of everyday life was assessed using the Strengths and Difficulties Questionnaire (SDQ). Children's basic touch interaction skills to operate digital devices and skills to use functions of digital devices were also measured.

Results: The results indicated that during the pandemic, children were more prosocial, experienced more problems in their peer relationships, and had better digital skills, but no differences were found in emotional symptoms; conduct problems; and hyperactivity between, before, and during the pandemic. The differences in digital skills were explained by the duration of children's media use.

Conclusions: Overall, our results suggest that the pandemic may have an immediate impact on children's socio-emotional behaviors and digital skills.

Trial registration: Registration type: OSF Preregistration.

Date registered: April 27, 2020.

Registration from osf.io/tfh4k.

(https://osf.io/c7p6b)

\section{Background}

A novel coronavirus, SARS-CoV-2, has now spread widely throughout the world. Due to the virus' high transmission rate, relatively long incubation period, and increased mortality rate in people with certain conditions (e.g., older people), the World Health Organization (WHO) has provided guidelines to help prevent the public from becoming infected with the virus (1). The clinical course of the coronavirus disease, COVID-19, appears to be relatively mild in children compared to other populations $(2,3)$, although infants were found to be at high risk of becoming severely or critically ill (4). Nevertheless, the effects of the societal changes implemented to decrease the likelihood of SARS-CoV-2 infection on children's cognitive, social, and emotional development are unknown. According to Bronfenbrenner (5), child development is a function of the interaction between several systems and includes culture, parental occupations, schooling, peer relationships, and parenting. Thus, changes in one system can directly or indirectly affect children's development. In the case of COVID-19, the pandemic can affect parents' work, 
children's schooling, and media use by both children and adults, which may in turn have significant effects on children's cognitive, social, and emotional development.

Common strategies to prevent infectious diseases include requesting people to stay at home, avoiding crowds or large gatherings, and practicing social distancing. Indeed, people in several countries have been prevented from going to work or school including kindergarten, and have fewer opportunities for inperson communication during the COVID-19 pandemic. The public health policy, referred to as quarantine, is characteristic of infectious diseases including the COVID-19 pandemic, compared to other negative events, such as natural disasters or terrorism. Although quarantine may play a major role in preventing the spread of COVID-19 cases (6), it may cause psychological problems such as depression, irritability, and anxiety $(7,8)$. Given that children may be more vulnerable to such situations than adults, we need to identify children's problems and, if necessary, support them accordingly. Several psychological studies have already examined the effect of the COVID-19 pandemic on children's mental health (e.g., depression, anxiety) (9-12). However, due to the nature of quarantine (the lack of social relationship with peers and teachers), children may have problems in socio-emotional behaviors (e.g., hyperactivity, prosocial behavior). It remains unclear whether the COVID-19 pandemic affects children's socio-emotional behaviors. One study in Italy and Spain reported that children experienced emotional and behavioral problems (e.g., difficulty concentrating) after the pandemic outbreak (11), but this study did not assess the population before the pandemic and thus the change in emotional problems could not be compared. Therefore, the present study examined differences in children's socio-emotional behaviors before and during the pandemic.

Moreover, even though children have problems in socio-emotional behaviors, supporting children inperson may be difficult because of the widespread disease. In that case, children can benefit from digital technology, such as online communication and learning. Indeed, it has been proposed that technology may be essential during a time of isolation and social distancing and can support the wellbeing of children (13). Although the online support is still limited presently, we believe that it may be potentially useful for children because even young children are capable of using digital technology. Furthermore, previous studies have shown that children can operate digital devices at the beginning of early childhood $(14,15)$. Children have the specific touch skills required to operate digital devices, such as the pinch, and tap gestures, and know how to utilize many of the functions of digital devices, such as video-calling (16).

Moreover, children may accelerate the development of digital skills under the COVID-19 pandemic; hence, it can be more beneficial to receive online support or online learning when they have problems in socioemotional behaviors. Many children cannot go to school due to the pandemic, and therefore may have increased opportunities to use digital devices, for instance, to receive online education. For example, the Chinese government started a large-scale, normal online education campaign during the pandemic (17). Consequently, children's skills in using digital devices may improve as they gain more experience. Therefore, in this pre-registered study, in addition to examining differences in children's socio-emotional behaviors, we assessed whether children showed differences in operating digital devices before and during the pandemic. 
In Japan, the first person infected with SARS-CoV-2 was identified in January 2020, and the number of infected people has since increased although the growth rate was lower than in many other countries [18]. On 27 February 2020, the government asked all schools across the country to close until March 2020 , and the vast majority of schools complied (but nursery schools did not). Schools started to re-open at the beginning of April 2020, but the government declared a state of emergency covering seven prefectures including Tokyo and Osaka on 7 April 2020. Thus, most schools in seven prefectures closed whereas about $80 \%$ of the kindergartens and half of the elementary schools in the other prefectures started to open on 10 April 2020 (19). Subsequently, the declaration to close schools was extended to all regions on 16 April 2020, and most schools in all prefectures closed until 6 May 2020. Thus, data for the during-pandemic sample was collected when most of the schools were closed and children had less time for schooling and meeting with friends.

\section{Data Collection}

For this study, we pre-registered our hypotheses, method, primary analyses, and sample size (https://osf.io/c7p6b). We conducted two cross-sectional studies in which we administered an internetbased survey to parents at two time periods, before and during the pandemic. Our before-pandemic sample comprised primary caregivers of children (ages $0-9$ ) who were randomly selected from the population of a database (Cross Marketing Inc. Tokyo, Japan). Sample size of each study includes 70 participants for each child's age. This required the total sample size of 840 in Study 1 (i.e., 420 for beforeand 420 for during-pandemic, respectively) and 1400 in Study 2 (i.e., 700 for before- and 700 for duringpandemic, respectively). To meet the sample size, additional participants were asked to answer the questionnaire because some were expected to be excluded for incorrect or inappropriate answers. Specifically, the data of before- and during-pandemic samples were collected as follows.

The before-pandemic sample completed the survey from 26-30 September 2019. A total of 1215 participants completed the questionnaire, but 293 participants were excluded, of which 255 participants incorrectly answered trap questions and 38 participants inappropriately answered questions (e.g., participants who chose " 1 " in a series of questions). Out of 922 participants (including 0 to 9 years of age), we assigned the first 70 participants in each age group (4 to 9 years of age) to Study 1 (a total of 420 participants) and first 70 participants in each age group ( 0 to 9 years of age) to Study 2 (a total of 700 participants). Sample characteristics of Study 1 and 2 are presented in Table 1, 2 and S1, 2.

Our During-pandemic sample was selected in the same way as our Before-Pandemic sample. No participants were the same between the Before- and During-pandemic period. The During-pandemic sample completed the survey from 28-30 April 2020. During recruitment, 1045 participants completed the questionnaire, but 152 participants were excluded, of which 81 participants incorrectly answered trap questions and 71 participants answered inappropriately. After assigning parents to the studies, we had a total of 420 parents in Study 1, and first 70 participants in each age group (0 to 9 years of age) were assigned to Study 2 (a total of 700 participants). 


\section{Study 1}

We assessed whether children's socio-emotional behaviors (emotional symptoms, conduct problems, hyperactivity, peer problems, and prosocial behavior) differed by their experience of the pandemic by comparing their socio-emotional behaviors before and during the pandemic. In terms of prosocial behavior, a previous study reported that experiencing a natural disaster (an 8.0 magnitude earthquake in China) affected children's prosocial behaviors (20). The researchers compared the prosocial behaviors of two groups: a group of 6- and 9-year-old Chinese children who lived near the epicenter of the earthquake before the disaster and a second group of Chinese children matched by age and school of attendance after the earthquake. The results suggested that 6-year-old children became more selfish whereas 9-yearold children became more prosocial immediately after the disaster. Although quarantine may impair children's socio-emotional behaviors, the COVID-19 pandemic may be different from the earthquake in several ways, the results from the previous study suggest that experiencing an adversity can have differential effects by age on children's prosocial behaviors.

Thus, we hypothesized that children would experience differences in their social relationships during the pandemic that would show the differences in their socio-emotional behaviors. Moreover, based on the previous study, we expected children's age to moderate the effect of the pandemic on prosocial behaviors. To assess the differences in social relationships, we assessed the duration of children's schooling, outside play, and lessons (e.g., music, dance).

\section{Study 1: Methods}

After assigning parents to the studies, we had a total of 420 parents in Study 1.

\section{Stimuli and procedure.}

The online questionnaire consisted of two parts. In the first part, parents were asked to complete background information about themselves and their children. In the second part, parents were given a questionnaire about their children's socio-emotional development and their social life.

Background information. In the first part, parents answered questions about their background. Background information included parental age, parental education, family size, children's age, children's sex, and children's sleep hours (when children get up and go to sleep). Parental education level was assigned a value from 1 to 5 (1=less than high school, 2=high school, $3=$ some college, 4=undergraduate degree, $5=$ graduate level).

Socio-emotional behaviors. In the second part, parents answered questions about their children's socioemotional behaviors. Children's socio-emotional behaviors in everyday life were assessed using the SDQ (Strengths and Difficulties Questionnaire) $[2,3](21,22)$. The SDQ is a screening measure of social, emotional, and behavioral functioning. The 25 -item SDQ is divided into five subscales, namely, emotional symptoms, conduct problems, hyperactivity, peer problems, and prosocial behavior. Emotion symptoms 
include five items, such as "Often complains of headaches, stomach-aches or sickness." Conduct problems include five items such as "Often fights with other children or bullies them." Hyperactivity includes five items such as "Restless, overactive, cannot stay still for long." Peer problems include five items, such as "Has at least one good friend." Prosocial behavior includes five items such as "Shares readily with other children, for example, toys, treats, and pencils." The parents answered whether each item applied to a child on a three-point scale from 0 "not true" to 2 "certainly true."

Social life. To assess the differences in children's social lives, questions were asked regarding the duration of time concerning children's schooling, children's outside play, and children's lessons (e.g., music, dance). We asked the number of days of children's schooling per week, and the average hours of outside play and lessons per day.

\section{Analytic plan}

Analyses were conducted in R (version 3.6.1). We conducted two analyses. First, we examined dependent variables that may be different before and during the pandemic. In our preregistration of the study, we planned to assess whether the period and children's age affected their social life and socio-emotional behaviors using a MANOVA. The analysis included period (Before-pandemic vs. During-pandemic) and age (0 to 9 ) as independent variables and durations of children's schooling, children's outside play and lessons, along with sub-scale scores for the SDQ as dependent variables. However, not all dependent variables were normally distributed, and we could not conduct the planned MANOVA. Instead, we conducted MANOVA within the framework of structural equation modelling (SEM). That is, we applied the MANOVA model to the data and estimated the parameters corresponding to the main effects using maximum likelihood estimation with robust (Huber-White) standard errors and a scaled test statistic that is (asymptotically) equal to the Yuan-Bentler test statistic using the "lavaan" package (23).

Second, we conducted a planned SEM analysis to assess the relationships between period and SDQ subscale scores, which were mediated by parents and children's social lives. Specifically, we used variables indicative of children's and parent's social lives if we found significant main effects of period in the preceding analyses. We used background information as control variables for the analyses if we found significant differences in the Before-pandemic and During-pandemic samples.

\section{Study 1: Results}

The descriptive data are reported in Table 1. Children's age in months, parental age, sex ratio (ratio of boys to girls), the number of family members, and parental education did not differ by period, Beforepandemic vs. During-pandemic. Children's sleeping time was higher During-pandemic than Beforepandemic $(\mathrm{t}(838)=-3.453, \mathrm{p}=.001, \mathrm{~d}=.24)$. Thus, Before-pandemic and During-pandemic samples generally matched. We included demographic variables as control variables in our subsequent analyses.

First, we assessed whether period and children's age affected children's socio-emotional behaviors and social lives. Period was significantly associated with children's peer problems $(\beta=0.264, p=.033)$ as well 
as durations of children's schooling $(\beta=-4.233, p<.001)$, children's outside play $(\beta=0.185, p=.001)$, and children's lessons $(\beta=-0.052, p=.032$ ) (Positive values represent increases during the pandemic compared to before the pandemic). We found a significant interaction between period and age in prosocial behavior $(\beta=0.080, p=.001)$. The effects of period were significant in $5-(\beta=1.000, p=.009)$ and 7-year-old $(\beta=0.843, p=.036)$ children. Children's prosocial behavior and peer problems are displayed as a function of age in Figure 1.

Next, we conducted SEM analyses to assess whether the effects of period and children's age on peer problems and prosocial behavior were mediated by differences in children's social lives. Specifically, we used durations of children's schooling, children's outside play, and children's lessons as mediation variables and children's sleeping time as a control variable (Figure 2). We selected the model that included direct paths between period and peer problems and between interaction and prosocial behavior $(X 2=62.304, \mathrm{RMSEA}=.047, \mathrm{CFI}=.966)$ because fit indices indicated that it provided a better fit to the data than a model without the direct path $((X 2=80.494$, RMSEA $=.053, \mathrm{CFI}=.952)$. In this model, period was positively $(\beta=0.491, p=.022)$ and negatively $(\beta=-2.843, p<.001)$ associated with durations of outside play and schooling, respectively. Interaction between age and period negatively associated with schooling $(\beta=-0.214, p<.001)$. In addition, the duration of play negatively associated with peer problems $(\beta=-0.261, p=.001)$. However, schooling was not significantly associated with prosocial behavior $(\beta=$ $0.113, p=.070)$.

Finally, we evaluated the mediation effects of outside play on the relationship between period and peer problems using Sobel tests. The estimated mediation effect of duration of outside play was significant ( $\beta$ $=-0.051, p=.01495 \% \mathrm{Cl}[-0.092-0.010])$. The estimated direct effect of period on peer problems was also statistically significant $(\beta=0.316, p=.01195 \% \mathrm{Cl}[0.0730 .558])$.

\section{Study 1: Discussion}

The results revealed that peer problems and prosocial behavior differed between before and during the pandemic; however, emotional symptoms, conduct problems and hyperactivity did not differ. Although there were no mediation effects on the relationship between period and prosocial behavior, we found an interaction effect between the pandemic and age in prosocial behavior. The results were partially consistent with our hypothesis that age may modulate the effect of the pandemic on prosocial behavior. Specifically, 4-year-old children scored equally before and during the pandemic, but older children showed more prosocial behavior during the pandemic compared to those before it. One possible interpretation for the increase in prosocial behavior was in-group favoritism. Items used to assess prosocial behavior included children's behavior towards in-group members, such as parents, siblings, or peers. Research on the behavioral immune system suggests that a pathogen infection can induce in-group favoritism and out-group aversion (24). The behavioral immune system refers to a motivational system that helps minimize infection risk by changing cognition, affect, and behavior to avoid infection with a pathogen. It has been consistently reported that the behavioral immune system in individuals at risk of infection facilitates stereotypes and prejudicial attitudes toward outgroup members and increases in-group 
favoritism, such as greater conformity to social norms and increased collectivism $(25,26)$. Such in-group favoritism may motivate cognitions and behaviors for the avoidance of novel parasites contained in outgroups and for the management of local infectious disease (27). Thus, children in this study may have increased prosocial behavior toward in-group members at the risk of pathogen infection to avoid infection by out-group members.

Children also showed more problems in their peer relationships during than before the pandemic. Although we found a mediation effect of outside play, the direct effect between the pandemic and peer problems was larger. Other factors, such as level of children's stress, can mediate the relationship between the pandemic and peer problems. Nevertheless, we need to be careful about the interpretation of the results because some items in peer problems (e.g., "tends to play alone") could be increased during the pandemic compared to before the pandemic unless children played with siblings as much as they used to play with peers. The increased scores did not necessarily mean the children were having trouble with peers. Overall, our results showed that some of the children's socio-emotional behaviors differed before and during the pandemic.

\section{Study 2}

In Study 2, we assessed whether children's digital skills and media use, such as their use of traditional media (TV and video), portable digital media (tablet PC and smartphone), and non-portable digital media (personal computer and gaming), differed before and during the pandemic. We focused on two digital skills that may have improved during early childhood: basic touch interaction skills to operate digital devices (hereafter, referred to as touch skills) and skills to use functions of digital devices (hereafter, function skills). Previous research reported that children develop the touch skills for tablet PC or smartphone, such as tap and drag, during early childhood $(16,28)$. For this study, we selected the following touch skills that may show age-related changes between ages 0 to 9: tap, double tap, one-hand drag, two-hand drag, enlarge/reduce screen, drawing with fingers, and drawing with digital pens. For function skills, we selected nine functions that previous studies (29) suggested that could be used during early childhood. These nine functions include viewing a picture, watching a video, taking a picture, recording a video, watching YouTube, enjoying apps, calling, video calling, and listening to music.

\section{Study 2: Methods}

\section{Stimuli and procedure.}

In addition to the background information in Study 1, parents were asked to complete the questionnaire about their own, and the children's media use and skills for operating digital devices.

Media use. Parents responded to questions about their own, and the children's media use. The questions were based on previous surveys of children's media use in Japan (29). First, parents completed questions about the children's media use. Specifically, parents reported the frequency of the media use per week 
(e.g., how often does your child use (MEDIA NAME) per week?) and per day (e.g., how many hours does your child use (MEDIA NAME) per day?). Media included TV, video, tablet computer, smartphone, nonportable computer, and non-portable games. Thereafter, parents also completed the questions about their own media use, and the questions were generally the same for parents and children. If the parents had partners (e.g., husband), they reported the average frequency of their and their partners' media use.

To assess the separate effect of each media on children's skills, we classified media use into traditional media (TV and video), portable digital media (tablet computer and smartphone) and non-portable digital media (non-portable computer and non-portable game). We calculated the average hours per day of children's use of each type of media for the analyses.

Skills to operate digital devices. Parents responded to questions about children's skills for operating digital devices. We focused on touch skills and function skills that may show age-related changes between ages 0 to $9(16,28)$. We selected seven touch skills: tap, double tap, one-hand drag, two-hand drag, enlarge/reduce screen, drawing by fingers, and drawing by pens. Parents were asked to evaluate each of the children's skills using a four-point scale (1. not at all, 2. very little, 3 . somewhat, 4 . to a great extent). In terms of function skills, we selected nine functions that can be used during early childhood based on the previous studies (29). The nine functions included viewing a picture, watching their own video, taking a picture, recording a video, watching YouTube, enjoying apps, call, video calling, and listening to music. Parents were asked to evaluate each of the children's skills using a four-point scale (1. not at all, 2. very little, 3. somewhat, 4 . to a great extent).

\section{Analytic plan}

Analyses were conducted in R (version $3.6,1)$ using the irtoys $(30)$ and lavaan packages. We conducted three analyses. First, we conducted item response theory analyses based on a two-parameter logistic model. That is, we converted the four-point scale data to a two-point scale data (we regarded 1 and 2 as zero, and 3 and 4 as one) and estimated item and ability parameters to separate the difficulty of each item in touch skills and function skills from each participants' ability for touch skills and function skills. Using a two-parameter logistic model, a correct response rate of participant $i$, whose ability is $\theta_{i}$, to item $j$ $P_{j}\left(\theta_{j}\right)$ are modeled by the following equation.

$$
P_{j}\left(\theta_{i}\right)=\frac{1}{1+e^{-\alpha_{j}\left(\theta_{i}-\beta_{j}\right)}}
$$

Here, $a_{j}$ and $\beta_{j}$ are parameters of item $j$ which represents item $j$ 's ability to discriminate $\theta_{j} s$ and difficulty.

Second, in our preregistration, we planned to conduct a MANOVA. The analysis includes period (Beforepandemic and After-pandemic) and age (0 to 9 ) as independent variables and the duration of digital devices use for children and parents, and the touch skills and function skills as dependent variables. However, as in Study 1, many dependent variables were not normally distributed, and we could not 
conduct the planned MANOVA. Instead, we conducted SEM analyses considering non-normal distribution using maximum likelihood estimation to assess whether period and age affected the duration of digital devices use for children and parents, and the touch skills and function skills.

Finally, we conducted SEM analysis to assess the relationship between period and skills for digital devices, which was mediated by the duration of digital media use. Specifically, we used variables assessing the duration of digital media for the mediation analyses if we found significant main effects of period in the second analyses. We used demographic characteristics as control variables for the analysis.

\section{Study 2: Results}

The descriptive statistics are reported in Table 2. Children's age in months, parental age, sex ratio (ratio of boys to girls), the number of family members and parental level of education did not differ between the Before-pandemic and During-pandemic samples. In terms of respondent's status (mother vs. father), the ratio of fathers to mothers was higher in the During-pandemic than the Before-pandemic sample $\left(\chi^{2}(1, N\right.$ $=1400)=6.116, p=.013)$. Children's sleeping time was also greater in the During-pandemic sample than Before-pandemic sample $(t(1398)=-2.412, p=.016, d=.13)$. There were no other significant differences in the Before-pandemic and During-pandemic samples. Thus, the Before-pandemic and During-pandemic samples generally matched. We included demographic characteristics as covariates in our subsequent analyses.

First, we conducted item response theory (IRT) analyses using a two-parameter logistic model to distinguish between the difficulty of each digital skill and a participant's ability. We reported the difficulty parameter and discrimination parameter of each item in touch and function skills in Table 3. Among the touch skills, tap was the easiest and the two-hand drag was the most difficult for children. In addition, viewing a picture was the easiest and video calling was the most difficult of the function skills.

Then, we assessed whether period and children's age affected each variable in children's media use and digital skills using MANOVA. The results revealed that period was significantly associated with touch skills $(\beta=0.235, p<.001)$ and function skills $(\beta=0.201, p<.001)$ as well as children's use of traditional media $(\beta=0.304, p<.001)$, children's use of portable digital media $(\beta=0.238, p<.001)$, children's use of non-portable digital media $(\beta=0.113, p=.005)$, parents' use of portable digital media $(\beta=0.229, p=$ $.007)$, and parents' use of non-portable digital media $(\beta=0.164, p=.025)$. In addition, age was positively associated with touch skills $(\beta=0.223, \mathrm{p}<.001)$ and function skills $(\beta=0.178, \mathrm{p}<.001)$ (Figure 3$)$.

Next, we conducted SEM analyses to assess whether period and age effects on touch and function skills were mediated by the duration of children's and parents' media use controlling for respondent status and children's sleeping time (Figure 4). The fit indices indicated that the model that included the direct path between period and touch skills and function skills $\left(X^{2}=2719.748\right.$, RMSEA $\left.=.074, \mathrm{CFI}=.936\right)$ was slightly worse than the model that excluded the direct path $\left(\chi^{2}=2692.540\right.$, RMSEA $\left.=.073, C F I=.937\right)$. Therefore, we selected the latter model as our final model. This model showed that period was positively associated 
with children's use of traditional media $(\beta=0.384, \mathrm{p}<.001)$, children's use of portable digital media $(\beta=$ $0.485, p<.001)$, children's use of non-portable digital media $(\beta=0.125, p=.003)$, and parents' use of portable digital media $(\beta=0.231 \mathrm{p}=.007)$. Moreover, touch skills were positively related to children's use of traditional media $(\beta=0.045, p=.016)$ and children's use of portable digital media $(\beta=0.210, p<.001)$. Function skills were also positively related to children's use of traditional media $(\beta=0.038, p=.031)$ and children's use of portable digital media $(\beta=0.207, \mathrm{p}<.001)$.

Finally, we evaluated the mediation effect between period and digital skills using Sobel tests. For touch skills, the estimated mediation effects on children's use of traditional media $(\beta=0.023, p=.00495 \% \mathrm{Cl}$ [0.008 0.039]) and children's use of portable digital media $(\beta=0.094, \mathrm{p}<.00195 \% \mathrm{Cl}$ [0.058 0.138]) were significant. Moreover, for function skills, the estimated mediation effects of children's use of traditional media $(\beta=0.020, \mathrm{p}=.00695 \% \mathrm{Cl}[0.0060 .034])$ and children's use of portable digital media $(\beta=0.095, \mathrm{p}$ $<.00195 \% \mathrm{Cl}[0.0510 .139])$ were significant.

\section{Study 2: Discussion}

There were several findings regarding children's digital skills. IRT analyses revealed the developmental sequence of touch and function skills, which was generally consistent with the results of previous behavioral studies $(16,28)$. In addition, the main analyses revealed that the durations of children's use of traditional and digital media was higher during than before the pandemic. However, the duration of parental use of digital media, but not traditional media, was higher during than before the pandemic. In addition, both touch and function skills were better during than before the pandemic. The results showed that the pandemic might have accelerated the children's skills for operating digital devices. Indeed, the touch and function skills in 5-year-olds during the pandemic exceeded those of 6 -year-olds before the pandemic.

Moreover, our mediation analyses revealed that the relationship between the pandemic and children's digital skills was mediated by children's use of portable digital media, but not parental use of digital media. Due to the pandemic, children could not go to school or kindergarten, and therefore children in this situation have more opportunities to use portable digital devices, such as for receiving online education. Consequently, children's skills in operating digital devices may be higher during the pandemic. However, parental use of devices did not have a significant impact on children's touch and function skills. Based on the results, we suggest that the pandemic provided children with more time to use digital devices, and children used such devices on their own or with their family, which may have facilitated the development of their touch and function skills.

\section{Conclusions}

Quarantine is characteristic of the COVID-19 pandemic, compared to other negative events, such as natural disasters or terrorism, and therefore we examined whether children have more problems in socioemotional behaviors during than before the pandemic. Our results in Study 1 showed that children 
exhibited better and worse socio-emotional behaviors during the COVID-19 pandemic compared to before the pandemic. Moreover, if they have problems in socio-emotional behaviors, children can benefit from support using digital technology, such as online communication and learning. Children might accelerate the development of digital skills under the COVID-19 pandemic because they may have increased opportunities to use digital devices. Results in Study 2 supported our hypothesis, showing that children exhibited better digital skills during compared to before the pandemic. Consequently, children can find it more beneficial to receive online support or online learning when they have problems in socio-emotional behaviors. To the best of our knowledge, this is one of the first studies to conduct a pre- and postassessment of the impact of the COVID-19 pandemic on children's behaviors.

One can argue that the parent questionnaire we used to assess digital skills of children may not be valid. However, our IRT analyses revealed that our questionnaire might be valid for assessing children's digital skills. Moreover, under circumstances in which COVID-19 is prevalent, it would be difficult to directly assess children's behaviors through experimental methodologies, especially with a large sample size. Although we assessed children's behavior using an online questionnaire, most of the available research did not utilize the same method of assessment both before and during the pandemic, which results in the difficulty of not having a valid comparison group. It is possible that parents' answers to the surveys can reflect differences in parents, not in children, and we need to be careful about the interpretations of the results. Nevertheless, we believe that web-based surveys may be one of the best methods for addressing the effects of the pandemic on child development.

Another limitation in this study was that we compared the different sample before and during the pandemic. We matched several types of background information that may have affected socio-emotional behaviors and digital skills across samples, but we need to conduct longitudinal research to examine how children change their behaviors across different time points. Moreover, it remains unclear whether the results from this population can be generalized to other populations, because the growth rate in the number of infected persons and deaths in Japan was lower than in other countries (18). Moreover, children's social-emotional development could be more severely impaired due to the pandemic, particularly if this difficult situation continues for a long time. Finally, we need to examine whether children can benefit from online support if they have problems in socio-emotional behaviors. Children can benefit from online communication with teachers or peers, and they can learn about how to manage their emotions and behaviors using online materials. Future research should address these issues.

\section{Declarations}

\section{Ethics approval and consent to participate}

The study was conducted in accordance with the principles of the Declaration of Helsinki and the procedure of the study was approved by the local ethics committee. Written informed consent (including study purpose, methodology, risks, right to withdraw, duration of the experiment, handling of personal 
information, and voluntary nature of participation) was obtained from all participating parents prior to administering the survey.

\section{Consent for publication}

This manuscript did not include any individual person's data

\section{Availability of data and materials}

The datasets supporting this article have been uploaded online.

https://doi.org/10.6084/m9.figshare.12375281.v1

\section{Competing Interests}

The authors declare no competing interests.

\section{Funding}

This research was supported by grants from JSPS to the first author.

\section{Author Contributions}

YM and NY developed the study concept. All authors contributed to the study design. Data collection was performed by YM. All authors performed the data analysis and interpretation. YM drafted the manuscript. $\mathrm{CS}, \mathrm{XM}$, and NY revised the manuscript. All authors approved the final version of the manuscript for submission.

\section{Acknowledgments}

We thank Chika Harada and Nobuhiro Mihune for the helpful comments on an earlier version of the manuscript.

\section{References}

1. World Health Organization. Coronavirus disease (COVID-19) advice for the public. Geneva: WHO; 2020. https://www.who.int/emergencies/diseases/novel-coronavirus-2019/advice-for-public. Accessed 29 Apr 2020.

2. Pathak EB, Salemi JL, Sobers N, Menard J, Hambleton IR. COVID-19 in children in the United States: Intensive care admissions, estimated total infected, and projected numbers of severe pediatric cases in 2020. J Public Health Manag Prac. Preprint at https://pubmed.ncbi.nlm.nih.gov/32282440/ (2020).

3. Wu Z, McGoogan JM. Characteristics of and important lessons from the coronavirus disease 2019 (COVID-19) outbreak in China: Summary of a report of 72314 cases from the Chinese Center for Disease Control and Prevention. 2020;323(13):1239-1242. 
4. Dong Y, Mo X, Hu Y, Qi X, Jiang F, Jiang Z, Tong S. Epidemiology of COVID-19 among children in China. 2020;145:e20200702.

5. Bronfenbrenner, U. The Ecology of Human Development. Harvard Univ. Press; 1979.

6. Hellewell J, Abbott S, Gimma A, Bosse N.I, Jarvis C I, RussellT W, et al. The Centre for the Mathematical Modelling of Infectious Diseases COVID-19 Working Group. Feasibility of controlling COVID-19 outbreaks by isolation of cases and contacts. The Lancet. Global Health. 2020;8:e488e496. doi:10.1016/S2214-109X(20)30074-7

7. Bai Y, Lin C-C, Lin C-Y, Chen J-Y, Chue C-M, \& Chou P. Survey of stress reactions among health care workers involved with the SARS outbreak. Psychiatr Serv. 2004;55: 1055-1057. doi:10.1176/appi.ps.55.9.1055

8. Sprang G, \& Silman M. Posttraumatic stress disorder in parents and youth after health-related disasters. Disaster Med Public Health Prep. 2013;7:105-110. doi:10.1017/dmp.2013.22.

9. Golberstein E, Wen H, Miller BF. Coronavirus disease 2019 (COVID-19) and mental health for children and adolescents. JAMA Pediatrics. Preprint at https://jamanetwork.com/journals/jamapediatrics/fullarticle/2764730 (2020).

10. Xie, X. et al. Mental health status among children in home confinement during the coronavirus disease 2019 outbreak in Hubei Province, China. JAMA Pediatrics. Preprint at https://jamanetwork.com/journals/jamapediatrics/fullarticle/2765196, (2020).

11. Orgilés M. et al. Immediate psychological effects of the COVID-19 quarantine in youth from Italy and Spain. PsyArXiv. Preprint at https://psyarxiv.com/5bpfz/ (2020).

12. Pisano L, Galimi D, Cerniglia L. A qualitative report on exploratory data on the possible emotional/behavioral correlates of COVID-19 lockdown in 4-10 years children in Italy. Preprint at https://psyarxiv.com/stwbn/. Accessed 13 Apr 2020.

13. Goldschmidt, K. The COVID-19 pandemic: Technology use to support the wellbeing of children. J Pediatr Nurs. 2020;53:88-90.

14. McClure ER, Chentsova-Dutton YE, Holochwost SJ, Parrott WG, Barr R. Look at that! Video chat and joint visual attention development among babies and toddlers. Child Dev. 2018;89(1):27-36.

15. Huber B, Highfield K, Kaufman J. Detailing the digital experience: Parent reports of children's media use in the home learning environment. Br J Educ Technol. 2018; 49(5):821-833.

16. Vatavu RD, Cramariuc G, Schipor DM. Touch interaction for children aged 3 to 6 years: Experimental findings and relationship to motor skills. Int J Hum Comp St. 2015;74:54-76.

17. Zhou L, Wu S, Zhou M, Li F. 'School's out, but class' on', The largest online education in the world today: Taking China's practical exploration during the COVID-19 epidemic prevention and control as an example. Best Evid Chin Edu. 2012;4(2):501-519.

18. COVID-19 coronavirus pandemic. https://www.worldometers.info/coronavirus/. (Accessed 14 May 2020). 
19. Ministry of Education, Culture, Sports, Science and Technology. Report of opening schools in the wake of the novel coronavirus pandemic in Japan. Available from https://www.mext.go.jp/content/20200413-mxt_kouhou01-000006421_1.pdf (2020).

20. Li Y, Li H, Decety J, Lee K. Experiencing a natural disaster alters children's altruistic giving. Psychol Sci. 2013;24(9):1686-1695.

21. Goodman, R. The Strengths and Difficulties Questionnaire: A research note. J Child Psychol Psychiatr. 1997;38(5):581-586.

22. Moriguchi Y, Shinohara I, Todo N, Meng X. Prosocial behavior is related to later executive function during early childhood: A longitudinal study. Eur J Dev Psychol. 2020;17(3):352-364.

23. Rosseel Y. lavaan: An R package for structural equation modeling. J Stat Softw. 2012;48(2):1-36.

24. Ackerman JM, Hill SE, Murray DR. The behavioural immune system: Current concerns and future directions. Soc Personal Psychol Compass. 2018;12(2):e12371.

25. Murray DR, Schaller M. Threat(s) and conformity deconstructed: Perceived threat of infectious disease and its implications for conformist attitudes and behavior. Eur $\mathrm{J}$ Soc Psychol. 2012;42(2):180-188.

26. Wu BP, Chang L. The social impact of pathogen threat: How disease salience influences conformity. Pers Individ Dif. 2012;53(1):50-54.

27. Thornhill R, Fincher CL. The parasite-stress theory of sociality, the behavioural immune system, and human social and cognitive uniqueness. Evol Behav Sci. 2014;8(4):257-264.

28. Aziz NAA, Batmaz F, Stone R, Chung P WH. Selection of touch gestures for children's applications. In 2013 Science and Information Conference. IEEE;2013.p. 721-726

29. Takaoka J. Actual situation and attitude of media use of parents and children. Child Sci. 2017;14:610.

30. Partchev I, Maris G. irtoys: A collection of functions related to item response theory (IRT). R package version $0.2 ; 2017$

\section{Tables}


Table 1. Descriptive Statistics for Study 1

\begin{tabular}{|c|c|c|c|c|}
\hline \multirow[t]{2}{*}{ Measure } & \multicolumn{3}{|c|}{ BEFORE $(\mathrm{N}=420)$} & \multirow{2}{*}{$\begin{array}{l}\text { DURING }(\mathrm{N}=420) \\
\mathrm{SD}\end{array}$} \\
\hline & Mean & SD & Mean & \\
\hline \multicolumn{5}{|l|}{ Parent Measure } \\
\hline Parent's age & 39.74 & 5.81 & 40.44 & 5.38 \\
\hline Number of family members & 3.99 & 0.94 & 4.04 & 1.05 \\
\hline Parental level of education & 3.22 & 0.89 & 3.17 & 0.91 \\
\hline \multicolumn{5}{|l|}{ Children Measure } \\
\hline Children's age in months & 83.37 & 20.37 & 83.95 & 20.90 \\
\hline Sleeping hours & 9.35 & 0.81 & 9.54 & 0.77 \\
\hline Days of schooling per week & 4.95 & 0.49 & 0.72 & 1.73 \\
\hline Hours of outside play per day & 0.64 & 0.67 & 0.83 & 0.89 \\
\hline Hours of lessons per day & 0.26 & 0.35 & 0.21 & 0.37 \\
\hline Conduct problems & 2.45 & 1.73 & 2.37 & 1.82 \\
\hline Emotional symptoms & 2.10 & 2.11 & 2.19 & 2.14 \\
\hline Hyperactivity & 4.03 & 2.19 & 4.07 & 2.42 \\
\hline Peer problems & 2.24 & 1.75 & 2.50 & 1.84 \\
\hline Prosocial behavior & 5.38 & 2.45 & 5.90 & 2.43 \\
\hline Categorical Measure \% & $\%$ & & & \\
\hline Children's sex (ratio of girls) & $50 \%$ & & $50 \%$ & \\
\hline Respondent (ratio of mother) & $91 \%$ & & $88 \%$ & \\
\hline
\end{tabular}


Table 2. Descriptive Statistics for Study 2

\begin{tabular}{|c|c|c|c|c|c|}
\hline \multirow[t]{2}{*}{ Measure } & & \multicolumn{2}{|c|}{$\operatorname{BEFORE}(\mathrm{N}=700)$} & \multicolumn{2}{|c|}{ DURING $(\mathrm{N}=700)$} \\
\hline & & Mean & SD & Mean & SD \\
\hline \multicolumn{6}{|l|}{ Parent Measure } \\
\hline \multicolumn{2}{|l|}{ Parent's age } & 37.57 & 6.22 & 37.76 & 6.20 \\
\hline \multicolumn{2}{|l|}{ Number of family members } & 3.87 & 0.97 & 3.91 & 1.03 \\
\hline \multicolumn{2}{|l|}{ Parental level of education } & 3.24 & 0.90 & 3.23 & 0.91 \\
\hline \multicolumn{2}{|l|}{ Hour of traditional media per day } & 2.81 & 1.67 & 2.94 & 1.79 \\
\hline \multicolumn{2}{|c|}{ Hour of portable digital media per day } & 2.06 & 1.50 & 2.29 & 1.66 \\
\hline \multicolumn{2}{|c|}{ Hour of non-portable digital media per day } & 1.24 & 1.11 & 1.41 & 1.45 \\
\hline \multicolumn{6}{|l|}{ Children's Measure } \\
\hline \multicolumn{2}{|l|}{ Children's age in months } & 59.38 & 34.52 & 59.91 & 34.75 \\
\hline \multicolumn{2}{|l|}{ Sleeping hours } & 9.53 & 0.90 & 9.64 & 0.82 \\
\hline \multicolumn{2}{|l|}{ Hour of traditional media per day } & 1.70 & 1.33 & 2.00 & 1.54 \\
\hline \multicolumn{2}{|c|}{ Hour of portable digital media per day } & 0.53 & 0.97 & 0.77 & 1.26 \\
\hline \multicolumn{2}{|c|}{ Hour of non-portable digital media per day } & 0.28 & 0.75 & 0.39 & 0.84 \\
\hline \multicolumn{2}{|l|}{ Touch skill $\left(\theta_{i}\right)$} & -0.09 & 0.89 & 0.09 & 0.93 Function skill \\
\hline \multirow[t]{2}{*}{$\left(\theta_{i}\right)$} & -0.1 & 0.09 & \multicolumn{3}{|c|}{0.96 Categorical Measure } \\
\hline & Percent & & & & \\
\hline \multicolumn{2}{|l|}{ Children's sex (ratio of girls) } & $50 \%$ & & \multicolumn{2}{|l|}{$50 \%$} \\
\hline \multicolumn{2}{|l|}{ Respondent (ratio of mother) } & $94 \%$ & & \multicolumn{2}{|l|}{$90 \%$} \\
\hline
\end{tabular}

Note. $\theta_{i}$ represent participants' ability as the results of item response theory analyses 
Table 3. Difficulty and discrimination parameters of each item using two-parameter logistic model.

\begin{tabular}{|c|c|c|}
\hline Touch skill & $\beta_{j}$ (Difficulty) & $\alpha_{j}$ (Discrimination) \\
\hline Tap & -0.354 & 4.153 \\
\hline Screen & -0.066 & 3.166 \\
\hline Drawing by pens & 0.023 & 2.372 \\
\hline Drawing by fingers & 0.052 & 2.725 \\
\hline Double tap & 0.089 & 4.388 \\
\hline One-hand drag & 0.143 & 5.392 \\
\hline Two-hands drag & 0.321 & 6.646 \\
\hline Function skills & $\beta_{j}$ & $\alpha_{j}$ \\
\hline Viewing a picture & -0.377 & 5.490 \\
\hline Watching a video & -0.346 & 5.107 \\
\hline Taking a picture & -0.334 & 5.118 \\
\hline Watching YouTube & 0.001 & 1.998 \\
\hline Recoding a video & 0.165 & 3.336 \\
\hline Enjoying apps & 0.189 & 2.569 \\
\hline Calling & 0.344 & 2.392 \\
\hline Listening to Music & 0.398 & 2.125 \\
\hline Video-calling & 0.739 & 2.268 \\
\hline
\end{tabular}

Note: $\alpha_{j}$ and $\beta_{j}$ are parameters of item, which represent item $j$ 's ability to discriminate participants and difficulty, respectively.

\section{Figures}



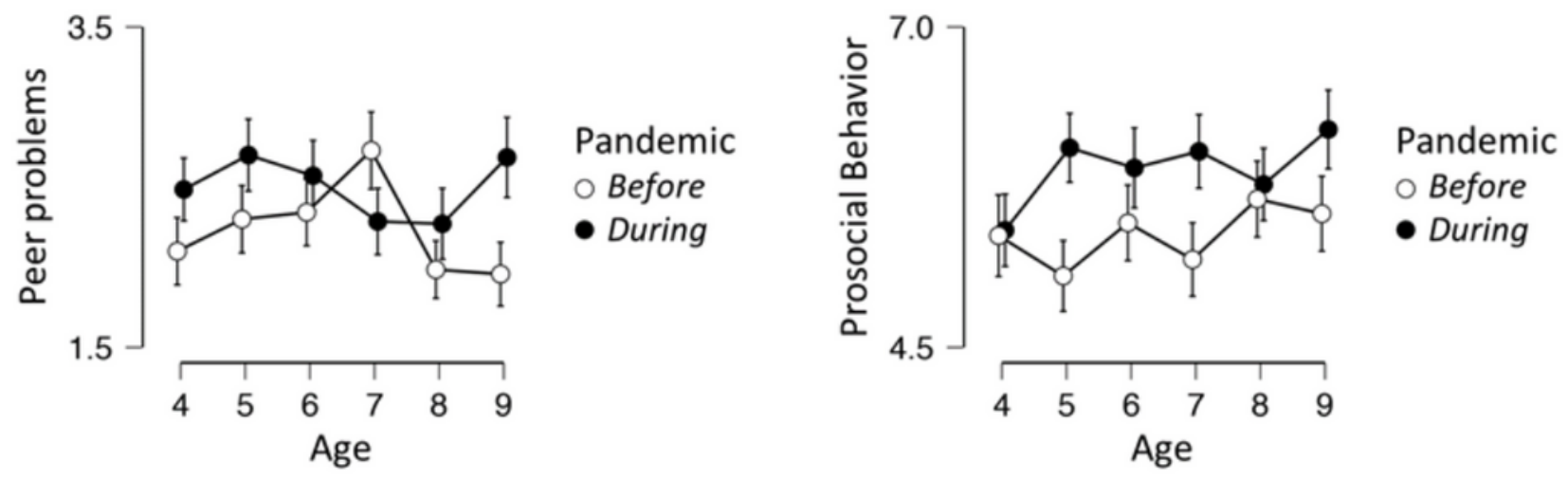

Figure 1

Children's prosocial behavior and peer problems as a function of age

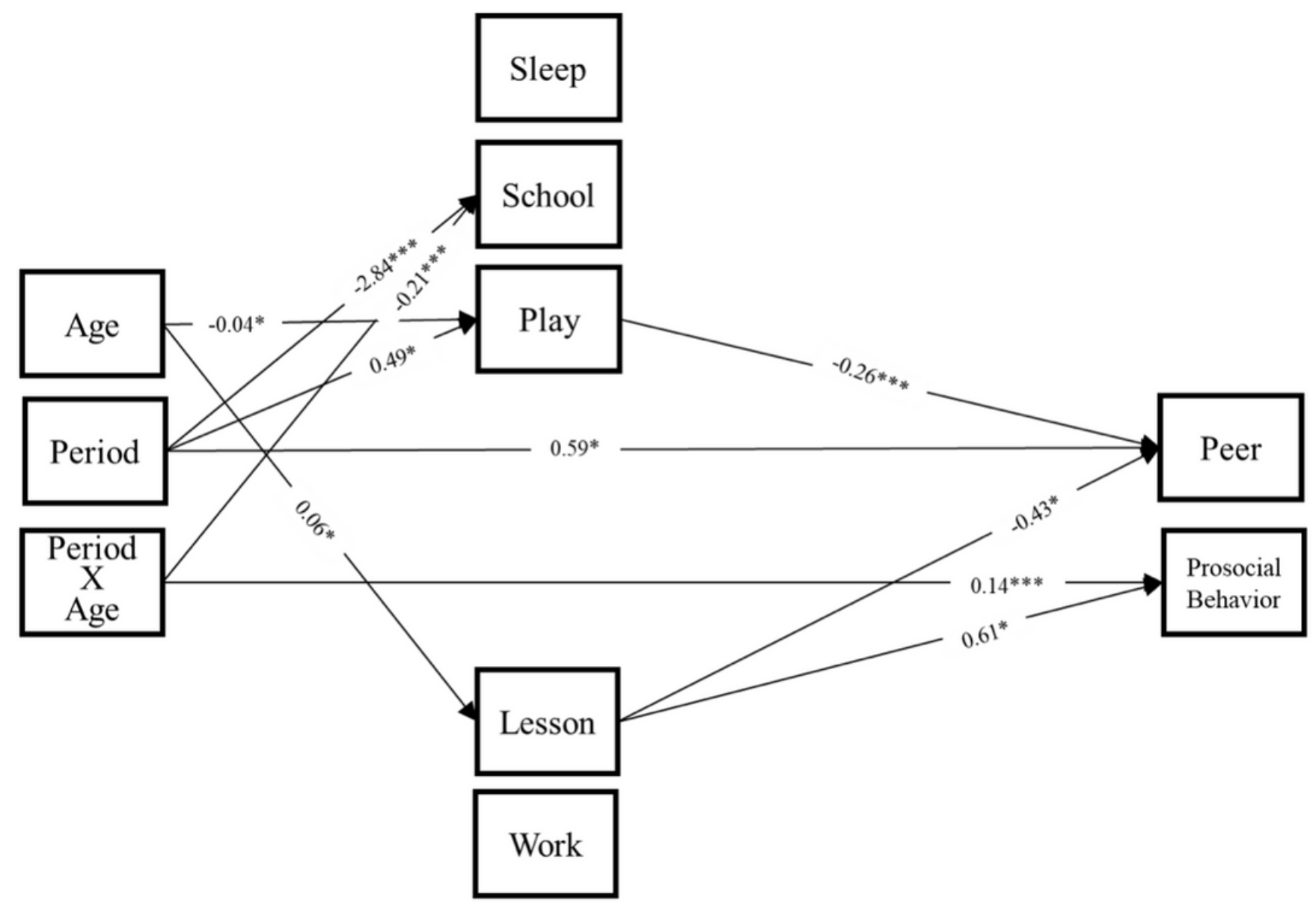

Figure 2 
Effects of pandemic (period), age and the interaction on children's prosocial behavior and peer problems. Sleeping time, children's sex, parental age, the number of family members, and parental education as control variables. For visibility, the non-significant paths, error variances, and covariance were removed and reported in Table S2. ${ }^{*} p<.05, * \star p<.01, * \star * p<.001$
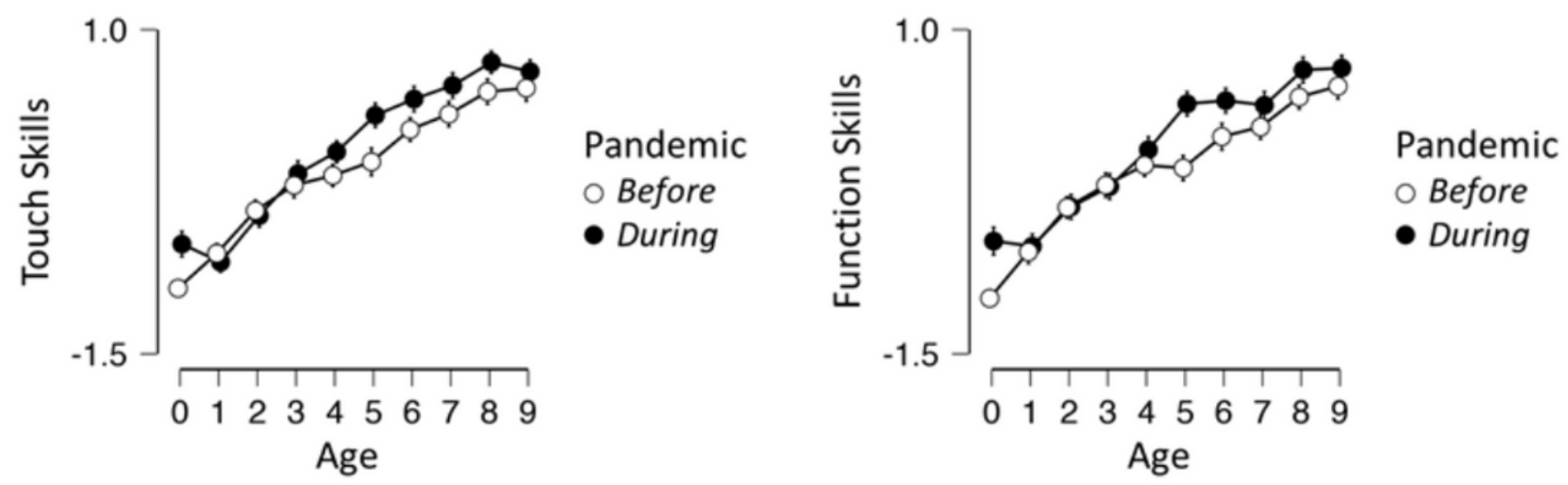

Figure 3

Children's touch skills and function skills as a function of age

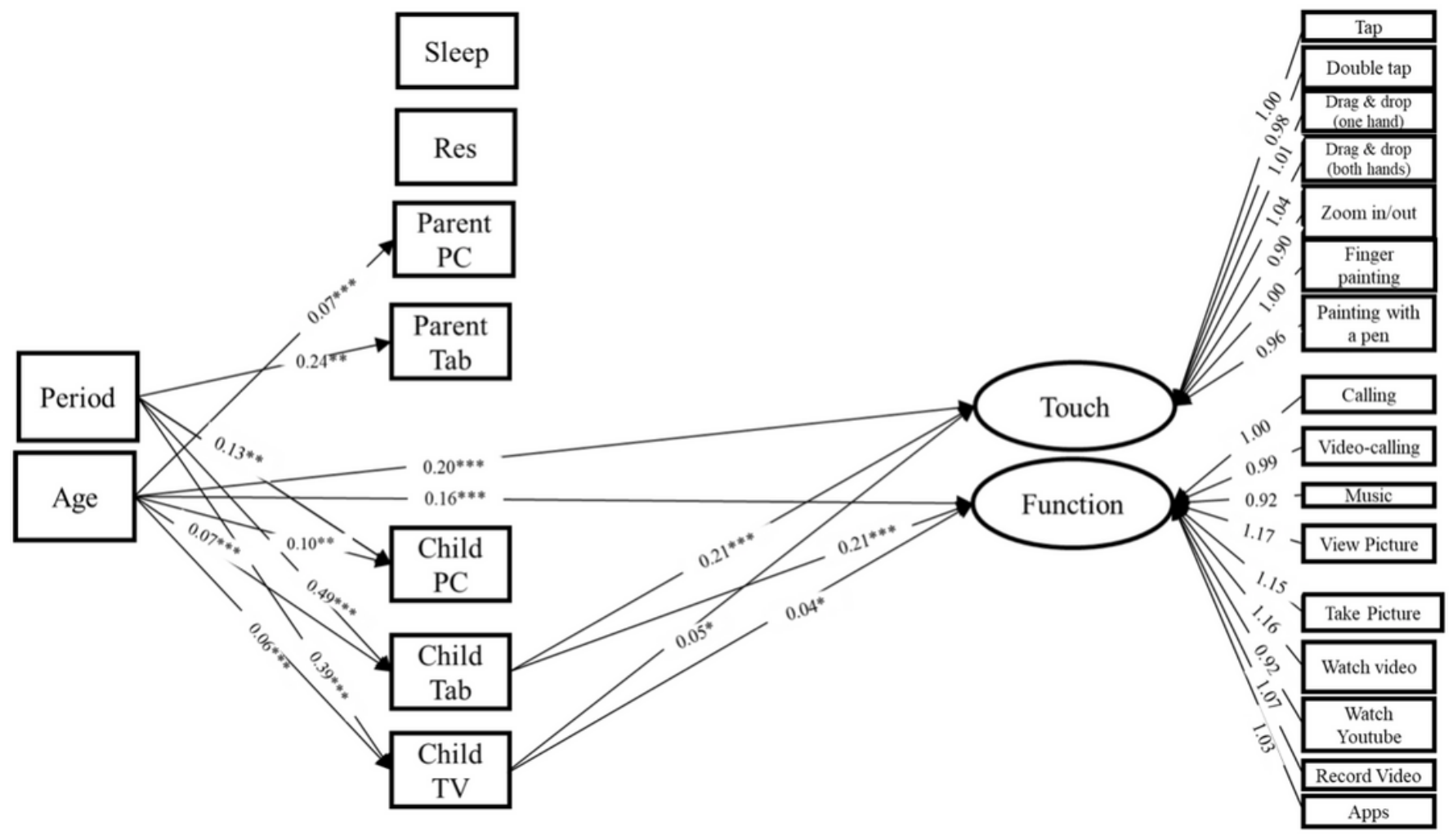


Effects of pandemic (period) and age on children's touch skills and function skills. Res represents respondent's status (mother vs. father), PC represents non-portable digital media, Tab represents portable digital media, and TV represents traditional media. Sleeping time, children's sex, parental age, the number of family members, and parental education are control variables. For visibility, the non-significant paths, error variances, and covariances were removed and reported in Table S3. ${ }^{*} p<.05, * \star p<.01, * \star * p<.001$

\section{Supplementary Files}

This is a list of supplementary files associated with this preprint. Click to download.

- SupTable.docx 